\title{
Analytical Expression for Sound Transmission Loss Calculation: An Improvement to the Existing Method after Singh and Katra
}

\author{
Iwan Yahya \\ The Iwany Acoustics \& Applied Physics Research Group (iARG), Physics Department, Faculty of Mathematics and Natural Sciences, \\ Sebelas Maret University, Jl. Ir. Sutami 36A Kentingan, Solo 57126, Indonesia \\ Correspondence should be addressed to Iwan Yahya, iwanyy@yahoo.com
}

Received 12 September 2009; Accepted 23 October 2009

Recommended by Fidel E. Hernandez Montero

\begin{abstract}
An analytical expression for measuring of sound transmission loss (TL) has been developed by using two microphones, an impedance tube and an impulse sound source as a proposed improvement to the existing procedure after Singh and Katra (1978). The calculation procedure is based on the autospectrum of short-time signals captured by the two microphones placed on two opposite positions from test sample while the sound source is on its surface. No spectral decomposition is required and the TL is calculated directly from the autospectrums of captured signals.
\end{abstract}

Copyright ( 2009 Iwan Yahya. This is an open access article distributed under the Creative Commons Attribution License, which permits unrestricted use, distribution, and reproduction in any medium, provided the original work is properly cited.

\section{Introduction}

Understanding of acoustic parameter and/or properties of material is very important in many applications. Nowadays it is ranging from tissue analysis for medical purposes to elastic properties analysis of material such as attenuation and TL in engineering applications. Researchers have developed methods and measurement techniques for these purposes. Such measurements simply categorized into two main groups: first, the standing waves method by using impedance tube with single microphone and single tone sound source; this method is inefficient due to its time consuming [ 1 , 2]; second, the spectral decomposition-based measurement, including a two-microphone impedance tube [3-5] and its four microphones improvement for TL measurement purposes [6-8]; the second group uses random noise instead of single tone sound source which is more efficient compared to the first group.

Another method for TL calculation is presented in this paper. The proposed method is an improvement to the previous one by Singh and Katra [9] and has been derived with two basic assumptions. (i) An impulse sound source is generated right on the sample surface while the two microphones are placed at two different opposite positions inside the tube. The microphone distance to the sample surface should be longer compared to the tube diameter to ensure that the propagating wave is plane wave. (ii) Tube is sufficiently long, so there are no reflected waves from the both ends; for special purposes the utilization of anechoic termination is needed.

\section{Existing TL Measurement Method by Singh and Katra}

In 1978, Singh and Katra introduced a procedure for measuring muffler characteristics based on impulse techniques as depicted in Figure 1.

The impulse sound is generated from loudspeaker at the end of the tube. For TL calculation purposes, the test sample is placed in certain position between both microphones. Upstream and downstream signals are captured by microphone number 1 and microphone number 2, respectively. A time window is set in an appropriate size to make sure that first detected signal captured properly and minimizing any part of reflected waves from both ends of the tube. The rule for this window sizing is expressed in detail by Singh and Katra. 


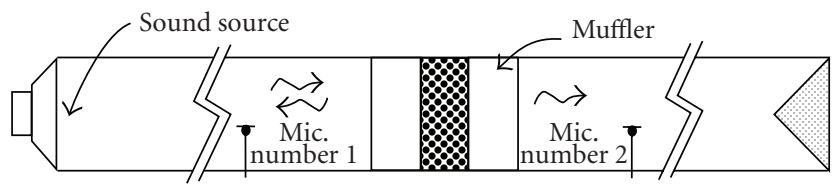

FIGURE 1: Setup for measuring muffler characteristics developed by Singh and Katra.

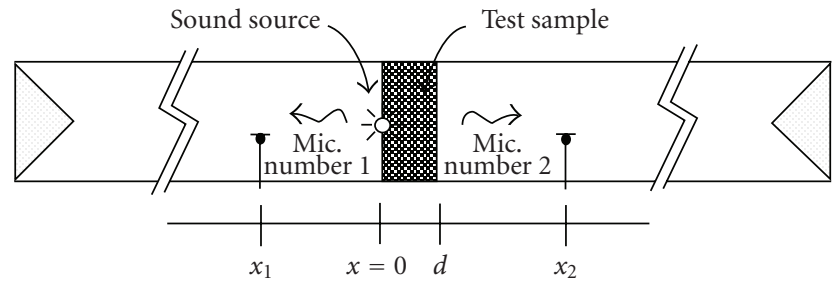

Figure 2: Proposed setup configuration for the new TL measurement.

The TL is then giving by

$$
\mathrm{TL}=10 \log \left\{\left(\frac{S_{12}}{S_{22}}\right)\left(\frac{S_{12}}{S_{22}}\right)^{*}\right\},
$$

where $S_{12}$ is cross power spectrum of signals captured by the microphones and $S_{22}$ is autopower spectrum of signal captured by microphone number two. The asterisk $(*)$ indicates complex conjugates of $S_{12}$ and $S_{22}$ ratios.

\section{Proposed Direct Calculation Procedure}

It happens that reflected waves are also captured by microphone number one, so TL in (1) was not obtained directly from incident and transmitted waves power spectrum.

To do this direct calculation, the modified configuration is depicted in Figure 2.

Short-time impulse sound is radiated from a source located directly on the left surface of the test sample. Time window is set in same manner as in Singh and Katra's method. Sound pressure values captured by the two microphones are given by

$$
\begin{aligned}
& p_{1}(x)=\sqrt{S_{11}}=p_{0} e^{\alpha x_{1}}, \\
& p_{2}(x)=\sqrt{S_{22}}=p_{d} e^{-\alpha\left(x_{2}-d\right)},
\end{aligned}
$$

where $\alpha=j k$ is the complex propagation constant of the waves in air inside the tube, $\sqrt{S_{11}}$ and $\sqrt{S_{22}}$ are autospectrum of the short-time captured signal by microphones number one and number two, respectively, and $p_{0}$ and $p_{d}$ are the sound pressure levels of the signals at $x=0$ and $x=d$, and in this case the $e^{j \omega t}$ terms were eliminated from the equations for simplification purpose.

An illustration of the short-time captured signal and its spectrum is depicted in Figure 3. Both microphones capture the signal in a same size window. Autospectrum of these captured signals is then used for TL calculation purposes.

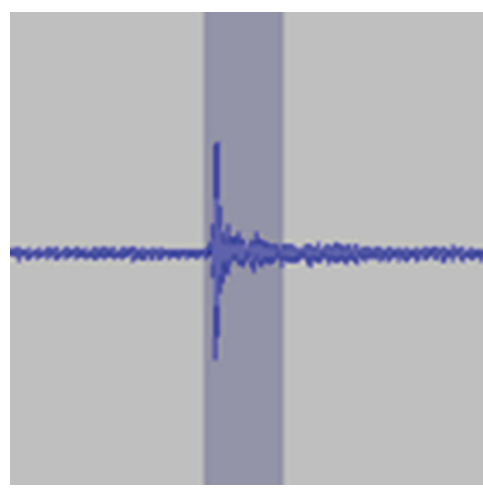

(a)

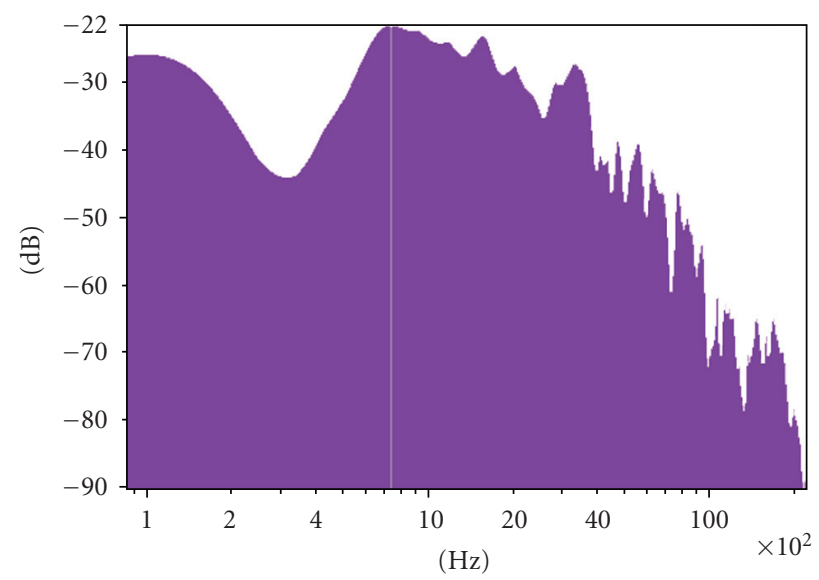

(b)

FIGURE 3: Illustration of first arrival truncation process.

From (2), one could write the expression for the sound pressure level on both surfaces of the test sample, for $x=0$ and $x=d$, as follows:

$$
\begin{aligned}
& p_{0}(x)=\sqrt{S_{11}} e^{-\alpha x_{1}}, \\
& p_{d}(x)=\sqrt{S_{22}} e^{\alpha\left(x_{2}-d\right)} .
\end{aligned}
$$

Let $x_{2}-d=-x_{1}$, then (4) becomes

$$
p_{d}(x)=\sqrt{S_{22}} e^{-\alpha x_{1}} \text {. }
$$

Expression for the sound pressure level in the test sample is given by

$$
p_{s}(x)=p_{0} e^{-\alpha_{s} x}, \quad 0 \leq x \leq d,
$$

where $\alpha_{s}$ is the complex propagation constant for the test sample. 
TL is then calculated by comparing the intensity of the incident waves and transmission waves using the following relation:

$$
\mathrm{TL}=10 \log \frac{W_{i}}{W_{t}}
$$

where

$$
\begin{aligned}
W_{i} & =\frac{p_{i}^{2}}{\rho c} A_{i}, \\
W_{t} & =\frac{p_{t}^{2}}{\rho c} A_{o},
\end{aligned}
$$

where $p_{i}$ and $p_{t}$ are given by (6) for $x=0$ and $x=d$, respectively, $\rho$ is the density of the air, and $c$ is sound velocity. $A_{i}$ and $A_{o}$ are cross sections of both sides of the tube.

By using the relation in (3), then (8) changes to

$$
W_{i}=\frac{p_{i}^{2}}{\rho c} A_{i}=\frac{S_{11} e^{-2 \alpha x_{1}}}{\rho c} A_{i} .
$$

Taking the boundary condition for $x=d$ and using (5) and (6) give $p_{t}=p_{s}(d)=p_{0} e^{-\alpha_{s} d}=\sqrt{S_{22}} e^{-\alpha x_{1}}$. Equation (9) could be rewritten as

$$
W_{t}=\frac{S_{22} e^{-2 \alpha x_{1}}}{\rho c} A_{o} .
$$

Since both sides of the tube have an equal cross-section, $A_{i}=A_{o}$, substitution of (10) and (11) into (7) gives the expression for sound transmission loss as

$$
\mathrm{TL}=10 \log \frac{S_{11}}{S_{22}} .
$$

Substituting (3) and (5) into (6) for $x=0$ and $x=d$, we get the expression for $\alpha_{s}$ as follows:

$$
\alpha_{s}=-\frac{1}{d} \ln \left(\frac{\sqrt{S_{22}}}{\sqrt{S_{11}}}\right)=-\frac{1}{d}\left[\ln \sqrt{S_{22}}-\ln \sqrt{S_{11}}\right] .
$$

Since this method requires only calculation of autospectrum of both truncated signals from the microphones, (12) and (13) show the effectiveness of the proposed method which is more simple in analysis compared to the previous calculation method by Singh and Katra, or even with the four-microphone technique.

Properly fitted anechoic termination is highly recommended to ensure that no parts of reflected waves are captured by the microphones. But this is not such a critical problem by the advance on surface microphone technology: by using a pair of surface microphones, a similar result could be obtained by placing the microphones properly on both surfaces of the test sample. An impulse sound source generated signal from the end of the tube or even the source placed on the sample surface, and TL could be derived directly from (6) with

$$
\begin{aligned}
& p_{i}=\sqrt{S_{11}}=p_{s}(0), \\
& p_{t}=\sqrt{S_{22}}=p_{s}(d) e^{-\alpha_{s} d} .
\end{aligned}
$$

It is obvious that (14) and (15) give similar results for TL and $\alpha_{s}$ as obtained from (12) and (13).

\section{Conclusion}

The proposed method is more efficient and faster compared to the existing procedure, even promising a better performance by the advance of new surface microphone technology. The ability of giving similar result to previous techniques is the advantage of the current proposed method.

\section{Acknowledgments}

The author would like to thank his family for their support and patience, and also his colleagues and students at the Physics Department.

\section{References}

[1] P. Dickens, J. Smith, and J. Wolfe, "Improved precision in measurements of acoustic impedance spectra using resonance free calibration loads and controlled error distribution," Journal of the Acoustical Society of America, vol. 121, no. 3, pp. 14711481, 2007.

[2] S. Sugie, J. Yoshimura, and H. Ogawa, "Absorption characteristics of fibrous material covered with perforated facing and film," Acoustical Science \& Technology, vol. 27, no. 2, pp. 87-96, 2006.

[3] R. Lanoye, G. Vermeir, W. Lauriks, R. Kruse, and V. Mellert, "Measuring the free field acoustic impedance and absorption coefficient of sound absorbing materials with a combined particle velocity-pressure sensor," Journal of the Acoustical Society of America, vol. 119, no. 5, pp. 2826-2831, 2006.

[4] A. F. Seybert, "Two-sensor methods for the measurement of sound intensity and acoustic properties in ducts," Journal of the Acoustical Society of America, vol. 83, no. 6, pp. 2233-2239, 1988.

[5] "Standard test method for impedance and absorption of acoustical materials using a tube, two microphones, and a digital analysis system," American Standard ASTM E 1050-98, 2003.

[6] Z. Tao and A. F. Seybert, "A review of current techniques for measuring muffler transmission loss," in Proceedings of the SAE Noise and Vibration Conference, 2003.

[7] A. R. Barnard and M. D. Rao, "Measurement of sound transmission loss using modified four microphones impedance tube," in Proceedings of the ASME Noise Control and Acoustics Division (Noise-Con '04), Baltimore, Md, USA, July 2004.

[8] O. Olivieri and J. S. Bolton, "Measurement of transmission loss of material using a standing wave tube," in Proceedings of the 35th International Congress on Noise Control Engineering (INTER-NOISE '06), Honolulu, Hawaii, USA, December 2006.

[9] R. Singh and T. Katra, "Development of an impulse technique for measurement of muffler characteristics," Journal of Sound and Vibration, vol. 56, no. 2, pp. 279-298, 1978. 

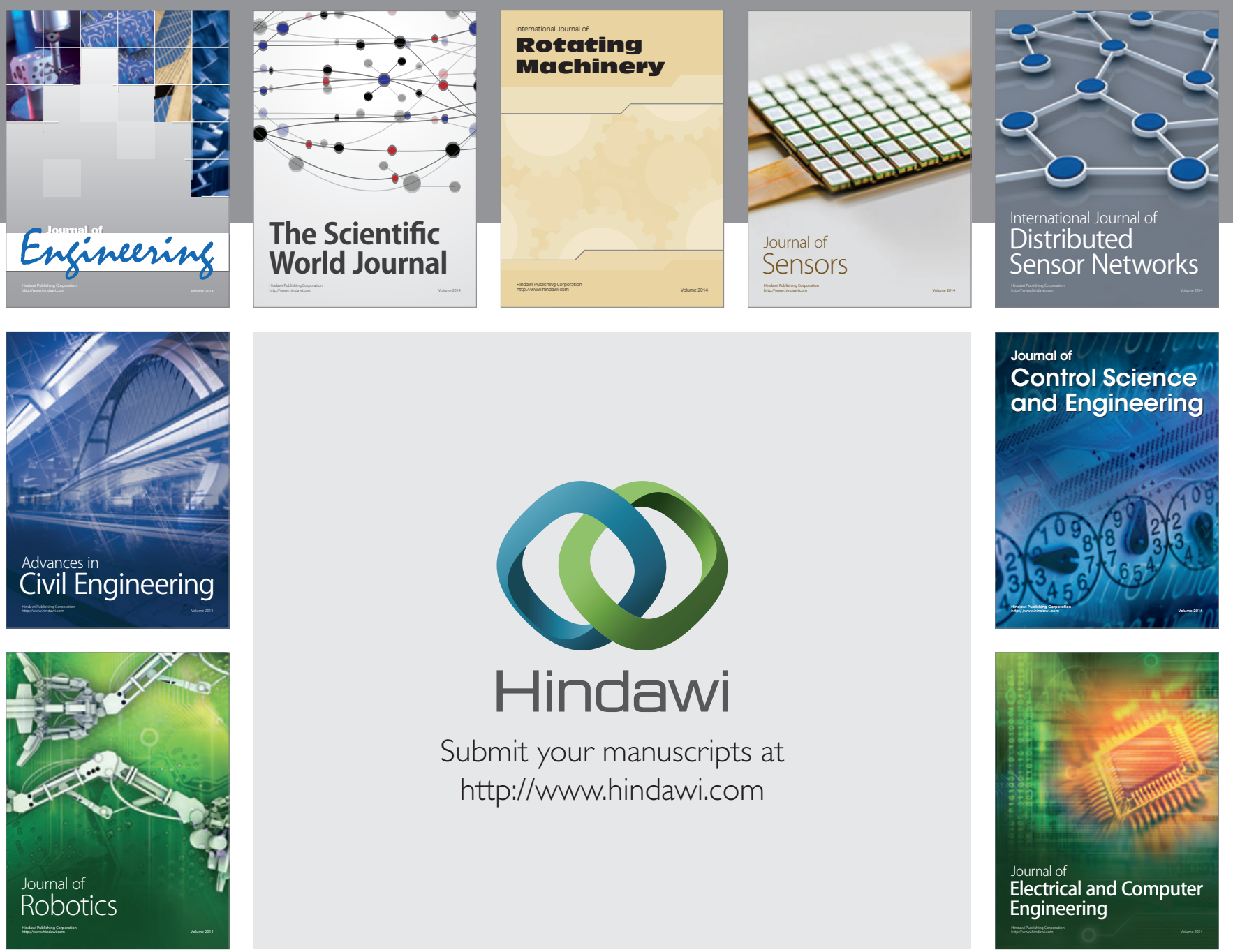

Submit your manuscripts at

http://www.hindawi.com
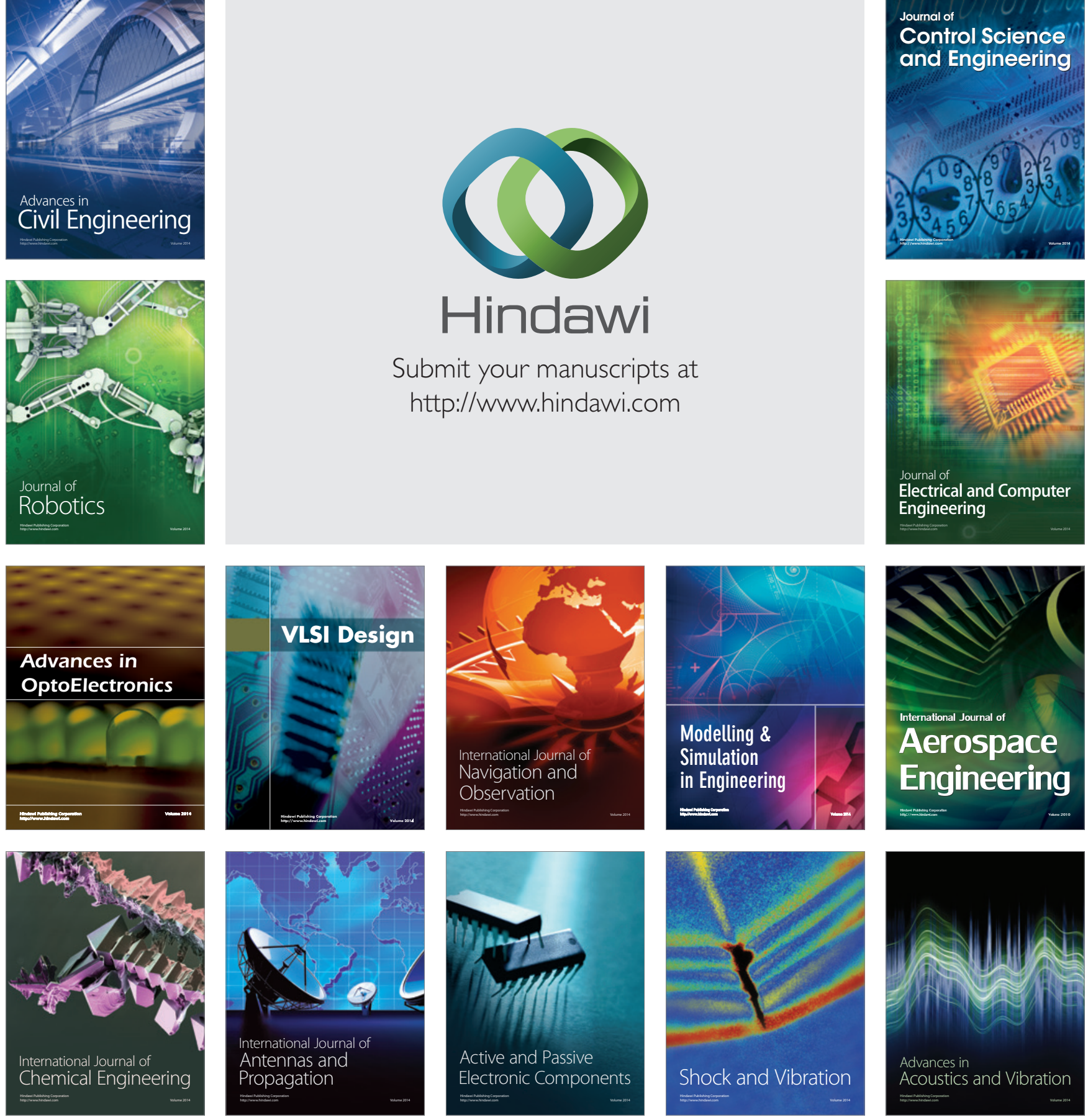